\title{
A Layered Multi-dimensional Description of Pilot's Workload Based on Objective Measures
}

\author{
Zhen Wang and Shan Fu \\ School of Aeronautics and Astronautics, Shanghai Jiao Tong University, \\ Shanghai, 200240, P.R. China \\ b2wz@sjtu.edu.cn
}

\begin{abstract}
Human factors have an important impact on aviation safety. The evaluation of pilot's workload is one of the most noteworthy human factors issues. After a brief overview of workload measurement techniques, a layered multi-dimensional description of workload is proposed, and the method is based on multiple objective measures. Heart rate, respiration, eye movements, control inputs and flight data are recorded in a simulated flight. The sensitivity and diagnosticity of several psychophysiological measurements are analyzed. Finally, a multi-dimensional pattern is constructed using the proposed method. The pattern can give a detailed description of pilot's workload throughout the flight.
\end{abstract}

Keywords: pilot's workload, layered multi-dimensional description, objective measures, simulated flight, workload pattern.

\section{Introduction}

Safety has always been the most considerable problem of civil aviation. After many years of effort, the reliability of the aircraft itself has been greatly developed. However, statistics shows that more than $60 \%$ of modern aircraft accidents are caused by human factors. In this situation, it's important to carry out human factors research in the cockpit to acquire information about human capability and limitations. Using this information in design, training and certification can increase the safety, comfort and efficiency of the aircraft [1].

One of the most important aspects of human factors research is to evaluate operator's workload. Universal definition of workload doesn't exist, but there is general agreement that operator's workload is a multi-dimensional construct. Megaw (2005) pointed out that workload is the interaction of task factors, operator response, operator performance and additional stressors [2].

The state of art workload measurement techniques are generally organized into three broad categories: task performance measures, subjective rating scales and psychophysiological measures [2-6].

Task performance measures evaluate operator's workload by considering the completion of the task. Task performance measures can be classified into two types: primary task performance measures and secondary task performance measures. Primary task performance measures overlook the operator's initiatives and effort, thus it's of poor validity. Secondary task performance measures require great care during the 
design of the secondary task in order to ensure that the secondary task compete for the same resource as the primary task. And secondary task performance measures are intrusive to primary task performance. Furthermore, task performance measures are only sensitive to the global changes of workload, their diagnosticity are limited.

Subjective rating scales use the operator's experience to evaluate workload. Some of the subjective rating scales such as NASA-TLX and SWAT have been widely used in different fields. Multi-dimensional subjective rating scales have good diagnosticity and are easy to apply. But subjective rating scales can't evaluate workload in real time. They are always correlated with task performance. Sometimes participants may forget the high workload they experienced. And the weighting procedures of these techniques are always cumbersome.

Psychophysiological measures use the psychophysiological reaction of the operator to reflect the workload imposed on them. Frequently used psychophysiological measurements include ECG, EOG, EEG, EMG, etc. These measures can evaluate workload in real time. Many measuring equipment are now portable and wireless, thus they would bring little intrusiveness and can be accepted by the participants. But the relation between psychophysiological measurements and workload are not clear. Some measurements have poor diagnosticity and may be easily affected by other factors e.g. ambience changes. And the equipment is always expensive.

In this paper, a workload description method is proposed based on objective measures. These measurements are categorized into different aspect. And workload is represented in a multi-dimensional way. It aims at providing a real time, valid and diagnostic tool to evaluate pilot's workload.

\section{Proposed Workload Description}

In order to cope with the problems of the existing workload measures, the following methods are proposed to describe pilot's workload.

\subsection{Hierarchical Relationship}

Since the relationship between psychophysiological measurements and workload is indirect. It can be assumed that there are some intermediate parameters between psychophysiological measurements and workload. These intermediate parameters could be explained as different dimensions of workload.

\subsection{Multi-dimensional Representation}

In the proposed method, it is assumed that workload can be decomposed into four primary dimensions. They are:

- "Cognitive activity", which represents pilot's activities in perception, information processing, decision making, etc.

- "Control activity", which represents pilot's input activities in the cockpit.

- "Effort and fatigue", which represents pilot's initiative effort in difficult situation.

- "Flight performance", which represents the result of the pilot's control. 


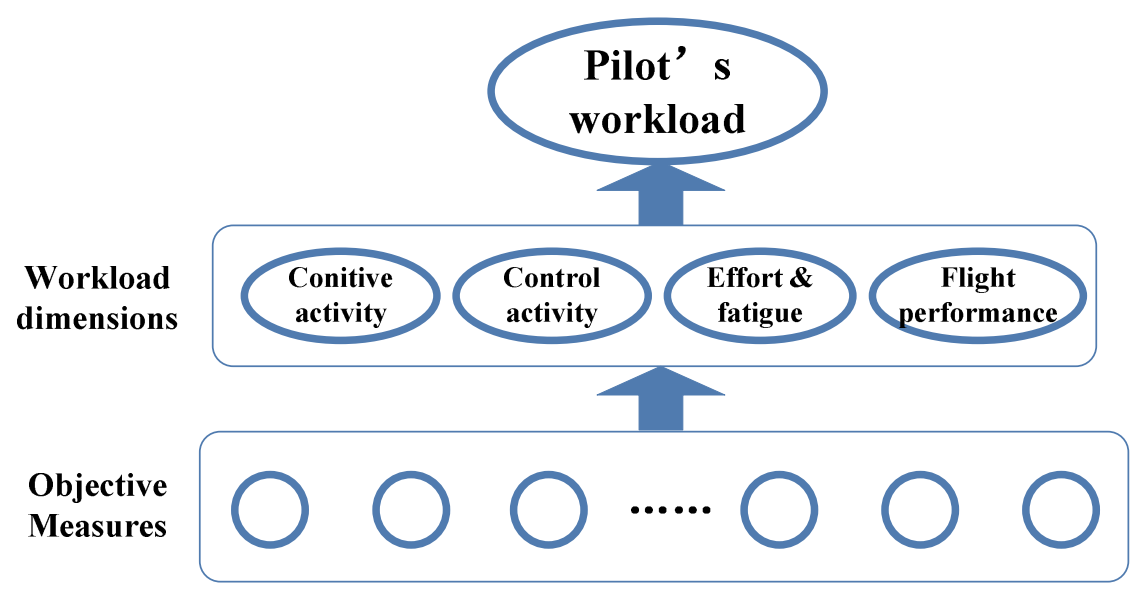

Fig. 1. Layered multi-dimensional description of pilot's workload

\subsection{Integrated Description}

Each primary dimension of workload can be integrated described by several objective measurements. Integrate different measurements which are sensitive to the same dimension can make them complement each other and can also reduce the interference of environment to single measurement.

By the above methods (i.e. hierarchical relationship, multi-dimensional representation and integrated description), the workload can be described by the structure as illustrated in Fig. 1.

\section{Experiment}

To empirically investigate the proposed workload description method, a simulated flight experiment has been conducted. During the experiment, several parameters have been objectively measured.

\subsection{Participants and Apparatus}

Eight students volunteered to take part in the flight simulation. They are aged from 23 to 29 , with the average of 26 . All of them are from the school of aeronautics and astronautics. They have basic knowledge about aviation and have been trained in simulated flight.

The experiment is carried out on a simulator with high fidelity. The simulator consists of two parts, the outside view and the cockpit. The outside view is simulated and projected on a cylindrical screen which has a diameter of about 8 meters. In the cockpit, the arrangements are referred to Boeing 777-200ER. There are control instruments and display instruments in the cockpit. The control instruments include the yoke, throttle, rudder pedal, flaps, landing gear, CDU and MCP. The display instruments include PFD, ND, EICAS, etc. 


\subsection{Procedure}

In the experiment, each participant is asked to fly a complete flight task 5 times. The flight task consists of take-off phase, cruise phase, approach and landing phase. The aircraft would take off from KSJC 30R. After passing 5 way points, it would be landed at KSFO 28R. The flight environment is simulated as in summer, at noon, sunny and no wind.

During the experiment, several parameters are recorded. Altitude, airspeed, position and instrument inputs are recorded by flight recorder. Blink, saccade, fixation and pupil diameter are recorded by SmartEye eye tracker. Heart rate, body temperature and respiration are recorded by BioHarness physiology monitoring system.

There is a training procedure before each experiment. Participant is asked to relax and fly a free flight. In the meantime, psychophysiological parameters, control activities are measured to acquire the baseline.

\section{$4 \quad$ Results}

Several typical tracings of the psychophysiological measurements are depicted in Fig. 2.

\section{Discussion}

\subsection{Psychophysiological Reaction}

The experimental results in Fig. 2 show that several psychophysiological measurements vary with the different phases of the flight.

Blink latency is longer during the take-off phase and the approach and landing phase. Realizing that during the take-off phase, the participants have to check the status of the systems carefully; during the approach and landing phase, the participants are asked to achieve a visual approach and landing, they have to frequently acquire the information about the flight, in order to adjust the flight path. Thus, it can be informed that when pilots pay more effort to concentrate on visual information, their blink latency may become longer $[8,10]$.

Fixation duration can reflect the time for a participant to acquire information. During the beginning of the take-off phase the fixation duration is longer, this may because that to check the systems status is very important, the participants pay more attention. During the last part of the approach and landing phase, pilots have to continuously monitor several critical parameters, e.g. altitude, airspeed, heading, attitude, etc. On the contrary, during the cruise phase, autopilot is engaged. Pilots are relatively more relaxed. They don't have to read a gauge accurately, and they glance at the whole cockpit and outside view. Thus, longer fixation duration can inform more cognitive activity.

Heart rate and respiration rate are higher when pilots have more control activities. This may be explained as that more activities require more energy. Researches indicate 


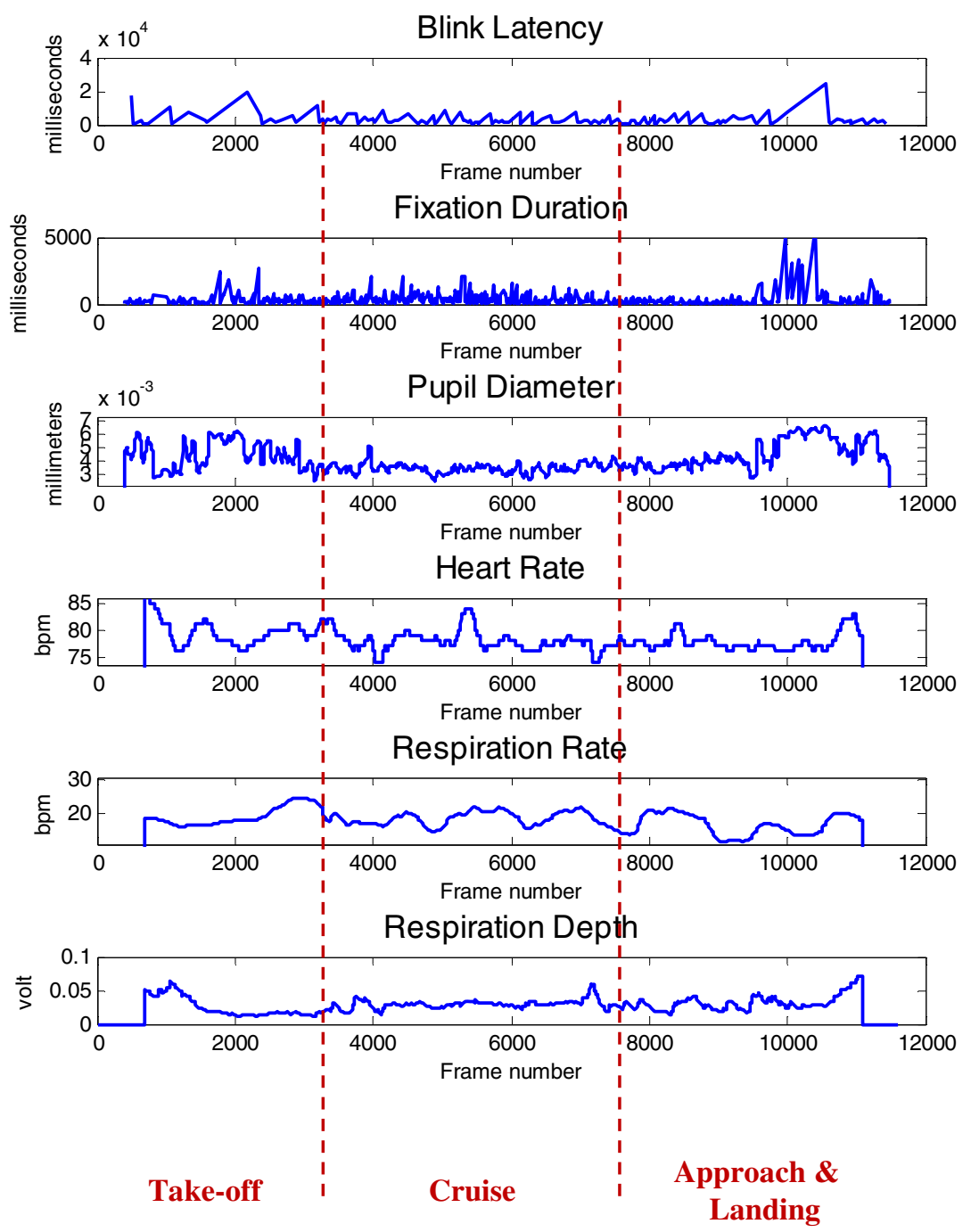

Fig. 2. Changes of psychophysiological measures throughout the flight

that heart rate, respiration and pupil diameter, etc. are associated with activities of the sympathetic nervous system [7-10]. When the task becomes harder or the operator feels more stressed, sympathetic nervous system accommodates heart and other organs to work harder.

Respiration depth seems to be sensitive to some particular events. When the participant discovers the performance falls, he would implement some adjustments. If these adjustments have good effects, the stress would be released and there often follows a deep breath. 


\subsection{Integrated Description of Workload Dimensions}

The psychophysiological measurements and other objective measurements can be classified into the four primary dimensions according to their diagnosticity. In each dimension, the following equations can be used to integrate the measurements.

In "cognitive activity" dimension, measurements can be integrated by the following equation:

$$
\text { cognition activity }=C_{1} \cdot \frac{t_{c g n}}{T_{c g n}}+C_{2} \cdot \frac{t_{f i x}}{T_{f i x}}
$$

Where

- $t_{c g n}$ is the cognitive time for a participant to perceive, process the information and make the decision before making a control. $t_{f i x}$ is the duration of each fixation. $T_{c g n}$ and $T_{f i x}$ are the baseline of cognitive time and fixation duration respectively, which are calculated from the training data.

- $C_{1}$ and $C_{2}$ are the weights to express the different contributions of cognitive time and fixation duration respectively. They are empirically set by the reliability of the measurements. In this paper, $C_{1}=0.6, C_{2}=0.4$.

In "control activity" dimension, measurements can be integrated by the following equation:

$$
\text { control activity }=K_{1} \cdot \frac{t_{c t r l}}{T_{c t r l}}+K_{2} \cdot \frac{m_{c t r l}}{M_{c t r l}}+K_{3} \cdot \frac{f_{c t r l}}{F_{c t r l}}
$$

Where

- $t_{c t r l}$ is the time for a participant to achieve a input by a control instrument. $m_{c t r l}$ is the magnitude of each input, represented by percentage of the instrument's extent. $f_{c t r l}$ is the frequency of the control activities. $T_{c t r l}, M_{c t r l}$ and $F_{c t r l}$ are the baseline of control time, control magnitude and control frequency respectively, which are calculated from the training data.

- $K_{1}, K_{2}$ and $K_{3}$ are the weights to express the different contributions of control time, control magnitude and control frequency respectively. They are empirically set to balance the different scale of the components. In this paper, $K_{l}=0.4, K_{2}=0.4$, $K_{3}=0.2$.

In "effort and fatigue" dimension, measurements can be integrated by the following equation:

$$
\text { effort \& fatigue }=E_{1} \cdot \frac{t_{b l}}{T_{b l}}+E_{2} \cdot \frac{h r}{H R}+E_{3} \cdot \frac{\text { pdiam }}{\text { PDiam }}+E_{4} \cdot \frac{\text { res }}{R E S}
$$

Where

- $t_{b l}$ is the blink latency. $h r$ is the heart rate. pdiam is the pupil diameter. res is the respiration rate. $T_{b l}, H R, P D i a m, R E S$ are the baseline of blink latency, heart rate, pupil diameter and respiration rate respectively. They are calculated from the training data. 
- $E_{1}, E_{2}, E_{3}, E_{4}$ are the weights to express the different contributions of blink latency, heart rate, pupil diameter and respiration rate respectively. They are empirically set by the reliability of the data. In this paper, $E_{1}=0.3, E_{2}=0.4, E_{3}=0.2, E_{4}=0.2$.

In "flight performance" dimension, measurements can be integrated by the following equation:

$$
\text { performance }=P_{1} \cdot e^{-(|\Delta s| \times 10)}+P_{2} \cdot e^{-(|a| \times 0.5)}
$$

Where

- $\Delta s$ is the deviation of the flight path. $a$ is the acceleration of the aircraft.

- $P_{1}$ and $P_{2}$ are the weights to express the different contributions of flight path deviation and acceleration respectively. They are empirically set to balance the scale of the components. In this paper, $P_{1}=0.6, P_{2}=0.4$.

\subsection{Workload Pattern}

After working out the values in the primary dimensions, another 6 secondary dimensions can be constructed. These secondary dimensions which represent the correlations between any of the two primary dimensions include "cognition-performance correlation", "control-performance correlation", "cognition-effort correlation", "control-effort correlation", "effort-performance correlation" and "cognition-control correlation". Note that, for the sake of simplicity and in order to display the multidimensional pattern into a flat plane, the "effort-performance correlation" and "cognition-control correlation" dimensions are omitted.

Each dimension has been scaled into the extent of 0 to 20 . Then an 8-dimension workload pattern is formed. In almost every moment of the flight, the workload pattern can be provided. This can give a very detailed evaluation of pilot's workload throughout the whole flight.

\subsection{Pattern Diagnosis}

The pattern's diagnosis is discussed by several typical cases.

The pattern in Fig. 3 (a) is a frequently appeared pattern during flight. It illustrates that the primary task of the pilot is monitoring the system. There are very few control activities, and flight performance is mainly influenced by the monitoring activities.

Pattern in Fig. 3 (b) illustrates that the pilot doesn't have many cognitive activities or control activities. After paying a little effort, a good performance can be achieved. It can inform that the pilot is currently performing in a well-designed flight deck system.

Pattern in Fig. 3 (c) shows that the pilot is now paying a lot of efforts and feels tired. This is mainly caused by heavy cognitive activities. In the meantime, few controls are made. It can inform that the pilot is trying hard to estimate the flight situation.

Pattern in Fig. 3 (d) shows a bad situation. The pilot is trying hard to estimate the conditions and there are many control activities, but the flight performance is still worse. 


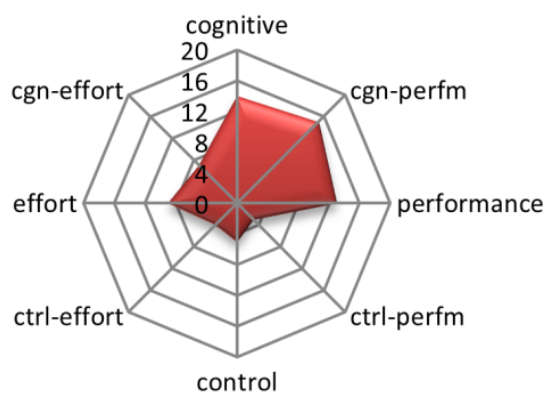

(a)

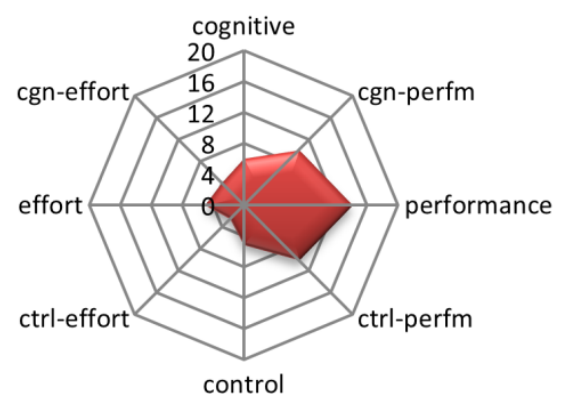

(b)

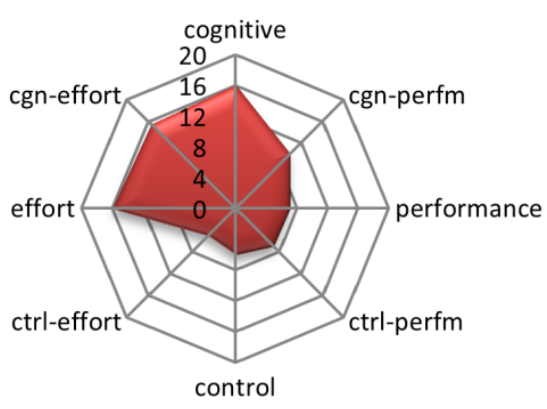

(c)

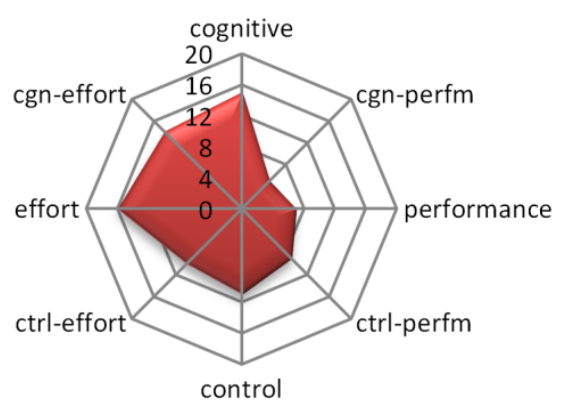

(d)

Fig. 3. Workload pattern cases

\section{Conclusions and Future Work}

In this study, several objective parameters are measured in a simulated flight. The experimental results show that blink latency, fixation time, pupil diameter, heart rate, respiration rate are sensitive to different aspects of workload. A layered multidimensional description of workload is proposed and the objective measurements are classified and integrated into four aspect of workload i.e. cognitive activity, control activity, effort and fatigue, flight performance. Furthermore, an 8-dimension workload pattern is formed. It can give a detailed and diagnostic evaluation of workload throughout whole flight. Yet, in the proposed method, several parameters are determined empirically. And the participants are not real pilots. The reliability of this method will be systematically examined in the future.

Acknowledgement. This research work was supported by National Basic Research Program of China-(973 Program No. 2010CB734103) and research program of Shanghai Jiao Tong University for innovation of post graduates (985 Program) with No. Z-413-006 under Grant TS0220741301. 


\section{References}

1. FAA System Safety Handbook, Chapter 17: Human Factors Principles \& Practices (2003)

2. Stanton, N.A., Salmon, P.M., Walker, G.H., Baber, C., Jenkins, D.P.: Human Factors Methods: A Practical Guide for Engineering and Design. Ashgate (2005)

3. Farmer, E., Brownson, A.: QinetiQ: Review of Workload Measurement, Analysis and Interpretation Methods. European organization for the safety of air navigation (2003)

4. Cain, B.: A Review of the Mental Workload Literature. (Tech. Rep. RTO-TR-HFM-121Part-II). Defence Research and Development Toronto, Toronto, Canada (2007)

5. Corwin, W.H., Sandry-Garza, D.L., Biferno, M.H., Boucek, G.P., Logan, A.L., Jonsson, J.E., Metalis, S.A.: Assessment of Crew Workload Measurement Methods, Techniques and Procedures. vol. I - Process, methods, and results (WRDC-TR-89-7006). Wright Patterson Air Force Base, OH (1989)

6. Harris, D.: Human Performance on the Flight Deck. Ashgate (2011)

7. Veltman, J.A., Gaillard, A.W.K.: Physiological Workload Reaction to Increase Level of Task Difficulty. Ergonomics 41, 656-669 (1998)

8. Wilson, G.F.: An Analysis of Mental Workload in Pilots During Flight Using Multiple Psychophysiological Measures. The International Journal of Aviation Psychology 12(1), 3-18 (2002)

9. Wickens, C.D., Hollands, J.G.: Engineering Psychology and Human Performance, 3rd edn. Prentice-Hall Inc., Upper Saddle River (2000)

10. Van Orden, K.F., Limbert, W., Makeig, S., Jung, T.P.: Eye Activity Correlates of Workload during a Visuospatial Memory Task. Human Factors 43(1), 111-121 (2001) 